\title{
LIGHT SCATTERING STUDY OF THE STATISTICAL PROPERTIES OF NEMATIC DIRECTOR FLUCTUATIONS
}

\author{
D. FRENKEL, M.J. VAN DORT and R.J.J. ZIJLSTRA \\ Fysisch laboratorium, Rijksuniversiteit Utrecht, P.O. Box 80000 , \\ 3508 TA Utrecht, The Netherlands
}

Received 11 September 1984

\begin{abstract}
Measurements of the photocount probability distribution and the half-open photocount timeinterval distribution of laser light scattered by some nematic liquid crystals are reported. It is found that the statistical properties of the scattered light are compatible with the assumption that director fluctuations behave as a Gauss-Lorentz process.
\end{abstract}

\section{Introduction}

Light scattering by nematic liquid crystals is caused by fluctuations in the dielectric tensor $\varepsilon$ of the fluid ${ }^{1}$ ). The most important contribution to fluctuations in $\varepsilon(r, t)$ is from fluctuations in the local nematic director $n(r, t)$ around its average value $n_{0}$. The relation between light scattering and director fluctuations has been analysed in detail by the Orsay Liquid Crystal Group ${ }^{2}$ ). These authors used standard linearized hydrodynamics to describe the decay of long-wavelength director fluctuations. The hydrodynamic theory predicts that director fluctuations at a given wavevector $q$ consist of two uncoupled modes, both of which decay exponentially. As the fluctuations in the dielectric tensor are to a good approximation linear in the director fluctuations, it follows that the scattered field too can be considered as the sum of two independent contributions. As a consequence, the fluctuations of the nematic director can be studied by dynamic light scattering (for a recent example, see ref. 3). In homodyne dynamic light scattering experiments the normalized autocorrelation function of the scattered intensity $g^{(2)}(\tau) \equiv\langle I(t) I(t+\tau)\rangle\left\langle\langle I\rangle^{2}\right.$ is measured. If the fluctuations responsible for light scattering are Gaussian, the intensity correlation function $g^{(2)}(\tau)$ is related to the normalized field correlation function $g^{(1)}(\tau) \equiv\left\langle E^{*}(t) E(t+\tau)\right\rangle /\left\langle E^{2}\right\rangle$ by

$$
g^{(2)}(\tau)=1+c\left|g^{(1)}(\tau)\right|^{2},
$$


where $c$ is a known ${ }^{4}$ ) spatial coherence factor. Eq. (1) is usually referred to as the Siegert relation (see e.g. ref. 5). Actually, the condition that fluctuations must be Gaussian is sufficient to guarantee the validity of eq. (1), but it is not a necessary condition. It has been argued by Tartaglia and $\mathrm{Chen}^{6}$ ) that even if the underlying hydrodynamic fluctuations are no longer strictly Gaussian, which happens for instance close to a critical point, deviations from eq. (1) are still unobservably small. A more sensitive quantity to study if one is interested in possible non-Gaussian effects is the photon-distribution function $P(n, T)$; the probability to detect $n$ scattered photons in an interval $T$. In contrast to the intensity correlation function, $P(n, T)$ is sensitive to all higher moments of the fluctuating variables. A closely related measurable quantity that is also sensitive to higher order moments is the distribution of time intervals between successive photocounts, $p_{\mathrm{i}}(t)$.

If the fluctuations responsible for light scattering behave as a Gauss-Lorentz process, the expressions for $P(n, T)$ and $p_{i}(t)$ are known in closed form (see e.g. Saleh ${ }^{5}$ ). The aim of the present study is to look for possible deviations from Gaussian behavior in the photon statistics of laser light scattered by nematic liquid crystals in the vicinity of the nematic-isotropic transition. Such deviations, if observed, would signal a breakdown of linearized hydrodynamics. Such a breakdown is conceivable because the nematic-isotropic transition is only weakly first order and, as one approaches this transition from the nematic side, some of the hydrodynamic transport coefficients vary rapidly with temperature $\left.{ }^{3.7}\right)$. Experiments similar to the ones described here were carried out by Chen et al $^{8}{ }^{8}$ ) in a study of critical fluctuations in a binary mixture.

\section{Experimental}

\subsection{Materials}

Light scattering experiments were performed on a variety of liquid crystalline materials, in particular: 5CB (p-cyano-p'-n-pentyl biphenyl; British Drug House); MBBA (p-methoxybenzylidene-p'-butylaniline; Riedel-de Haen), and DIBAB (p,p'-di-n-butylazoxybenzene; synthesized following ref. 9). All commercially obtained materials were used without further purification, DIBAB was recrystallized as described in ref. 9. The scattering cell had a thickness of $\approx 30 \mu \mathrm{m}$. Most liquid crystalline samples were prepared with planar alignment, except for some of the DIBAB samples which were made homeotropic.

\subsection{Light scattering setup}

The essentials of the light scattering setup have been described elsewhere ${ }^{3}$ ). 
Here we just mention those aspects of the experimental configuration that were different from the situation described in ref. 3. Scattered photons were detected by a fast photomultiplier (EMI $9863 \mathrm{~KB} / 100$ ). The photocounts were analysed by a Malvern-K7026 digital correlator system. For the analysis of time-interval statistics we used an Elscint TAC-N-1, time-to-amplitude converter coupled to a TRACOR-Northern TN1700 pulse height analyser. To be more precise, the TAC-N-1 was triggered by a pulse from a pulse generator and stopped by the photocount pulse. As a consequence, we do not measure $p_{\mathrm{i}}(t)$, the closed time-interval distribution, but $p_{\mathrm{f}}(t)$, the half-open time-interval distribution $\left.{ }^{5}\right)$. For a Gauss-Lorentz process the analytic expression for $p_{i}(t)$ is known too $\left.{ }^{5}\right)$. Both the Malvern K7026 and the TN1700 were micro-processor controlled. Further data analysis was performed on a PDP 11/70 minicomputer. In order to ensure good coherence of the scattered light, small pinholes $(\phi=100 \mu \mathrm{m})$ were placed close to the scattering cell and close to the detector, at a distance of $0.30 \mathrm{~m}$.

\subsection{Corrections}

Two experimental factors that give rise to small but systematic errors in the experimental results could not be eliminated and hence had to be corrected for. The first is the effect of residual stray light and dark counts, the second the effect of the non-ideality of the photomultiplier tube. Both will be discussed below.

\section{1) Stray light and dark counts}

Most of the stray light is eliminated because all light to the detector passes through an analyser with its optical axis perpendicular to the polarization of the incident light. However, some depolarized scattering of laser light occurs at the glass-nematic interface and this source of stray light could not be eliminated. In addition, even in the absence of stray light, dark counts are detected at a constant rate of approximately $40 \mathrm{~Hz}$ (for the EMI 9863 at room temperature). Photocounts due to stray light and dark counts behave as a Poisson process. Hence the photocount statistics are those of a combined Poisson-Gaussian-Lorentz process. Typically the intensity of the uncorrelated Poisson light was less than $2 \%$ of the total intensity. Once the relative intensity of the uncorrelated light is known $P(n, T)$ and $p_{\mathrm{f}}(t)$ can be evaluated analytically $^{5}$. However, as we do not know the level of stray light a priori we used the fraction of uncorrelated Poisson light as a variable in our final non-linear least squares fitting. We always found this fraction to be small, typically $\approx 1 \%$, except for the homeotropic DIBAB sample, where the signal itself was rather weak; here the fraction of uncorrelated photocounts could be as high as $15 \%$. 


\section{2) Non-ideal photomultiplier tube}

Two properties of the PM-tube should be taken into account when analysing the statistics of photocounts. The first is the finite deadtime $\tau_{\mathrm{D}}$ of the detector and the second the small but finite probability of afterpulsing. Because the deadtime turns out to be very small $\left(\approx 1.6 \times 10^{-8} \mathrm{~s}\right)$ and the probability of afterpulsing is quite small $\left(P_{\mathrm{A}} \approx 7.7 \times 10^{-5}\right)$, corrections for these effects were only needed for the photocount distribution function $P(n, T)$ and for the computation of the "real" photocount rate. The relation between the average observed count rate $\bar{n}_{0}$ and the rate $\bar{n}$ that would be observed if no deadtime and afterpulsing effects were present is given by ${ }^{10}$ )

$$
\bar{n}_{0}=\bar{n}\left(1-\alpha \tau_{\mathrm{D}} \bar{n}+P_{\mathrm{A}}\right),
$$

where $\alpha=1$ for Poisson light and $\alpha=2$ for Gaussian light. Here we have assumed that both $\tau_{\mathrm{D}} \bar{n}$ and $P_{\mathrm{A}}$ are $\ll 1$. To the same level of approximation the observed photocount distribution function $P_{\mathrm{o}}(n, T)$ is related to the ideal distribution $P(n, T)$ by

$$
\begin{aligned}
P_{\mathrm{o}}(n, T)= & P(n, T)+n \frac{\tau_{\mathrm{D}}}{T}\{(n+1) P(n+1, T)-(n-1) P(n, T)\} \\
& +P_{\mathrm{A}}\{(n-1) P(n-1, T)-n P(n, T)\} .
\end{aligned}
$$

Here $P(n, T)$ is the photocount distribution for a combined Poisson-GaussLorentz process as referred to above. Several methods exist to determine $P_{\mathrm{A}}$ and $\tau_{\mathrm{D}}{ }^{10}$ ). We made use of the very high speed of the correlator to measure both quantities directly by analysing the intensity correlation function of white light from an incandescent source. If deadtime effects and after pulsing were absent this correlation function should be perfectly flat, even at the highest time-resolution of the correlator $\left(\Delta t=20 \times 10^{-9} \mathrm{~s}\right)$. The correlator is constructed such that it can register at most 1 photocount in every "bin" of $\Delta t=20 \mathrm{nsec}$. Now suppose the deadtime of the PM tube is $t_{\mathrm{D}}<\Delta t$. If a photocount arrives somewhere in bin " $n$ " and another would arrive somewhere in bin " $n+1$ " there is a finite probability that the detection of this second photocount is blocked due to the PM deadtime. This blocking probability is equal to $P_{\mathrm{B}}=t_{\mathrm{D}}^{2} /\left(2 \Delta t^{2}\right)$. But if the second photocount would arrive in bin " $n$ " it will certainly be blocked because the correlator cannot register two counts per bin. Hence the effective dead-bins of the combined correlator-PM tube system is given by

$$
\begin{aligned}
T_{\mathrm{D}} & =(\Delta t)^{-1} \int_{0}^{\Delta t}\left(\Delta t-t^{\prime}\right) \mathrm{d} t^{\prime}+(\Delta t)^{-1} \int_{0}^{t_{\mathrm{D}}}\left(t_{\mathrm{D}}-t^{\prime}\right) \mathrm{d} t^{\prime} \\
& =\frac{1}{2} \Delta t+\frac{1}{2} t_{\mathrm{D}}^{2} / \Delta t=\Delta t\left(\frac{1}{2}+P_{\mathrm{B}}\right) .
\end{aligned}
$$


In eq. (4) $\Delta t$ is known $\left(\Delta t=20 \times 10^{-9} \mathrm{~s}\right)$ and $P_{\mathrm{B}}$ can be measured because this blocking will depress the apparent value of the first bin of the intensity correlation function of white light by a factor $1-P_{\mathrm{B}}$. Afterpulsing will have the effect of increasing the first bin of this intensity correlation function and, to some extent, subsequent bins. The expression for the value of the first bin of the intensity correlation function is

$$
g^{(2)}(\Delta t)=1-P_{\mathrm{B}}+P_{\mathrm{A}}^{(1)} / \bar{n}
$$

where $\bar{n}$ is the average number of counts per bin (which is typically $\ll 0.01$ ) and $P_{\mathrm{A}}^{(1)}$ is the probability of afterpulsing in the first bin. Clearly, by measuring $g^{(2)}(\Delta t)$ as a function of $1 / \bar{n}$, both $P_{\mathrm{B}}$ and $P_{\mathrm{A}}^{(1)}$ can be determined. For the next few bins only afterpulsing contributes to $g^{(2)}(l \Delta t)(l=2,3)$ :

$$
g^{(2)}(l \Delta t)=1+P_{\mathrm{A}}^{(l)} / \bar{n}
$$

The total afterpulsing probability is $P_{\mathrm{A}}=\Sigma_{l} P_{\mathrm{A}}^{(l)}$. We find that under normal operating conditions our PM tube has a deadtime

$$
\begin{aligned}
& t_{\mathrm{D}}(15.5 \pm 0.1) \times 10^{-9} \mathrm{~s}, \quad \text { while } \quad P_{\mathrm{A}}^{(1)}=(5.4 \pm 0.2) \times 10^{-5} \\
& P_{\mathrm{A}}^{(2)}=(1.9 \pm 0.1) \times 10^{-5} \quad \text { and } \quad P_{\mathrm{A}}^{(3)}=(0.35 \pm 0.06) \times 10^{-5}
\end{aligned}
$$

Hence $P_{\mathrm{A}}=(7.7 \pm 0.3) \times 10^{5}$. The effective deadtime of the combined correlator-PM tube system is found from eq. (4): $T_{\mathrm{D}}=(16.0 \pm 0.1) \times 10^{-9} \mathrm{~s}$. These values for $P_{\mathrm{A}}$ and $T_{\mathrm{D}}$ were used in the subsequent data analysis.

\section{Results and analysis}

All light scattering experiments were carried out with the polarization of incident and scattered radiation chosen such that only a single hydrodynamic mode, viz. the "twist-bend" mode ${ }^{2}$ ) was responsible for light scattering. In this case we should expect the intensity correlation function to consist of a single exponential. This was indeed always observed. To be more precise, the experimental single-clipped intensity correlation function never deviated significantly from a single exponential with a decay time $\tau / 2$. From these exponentials we determined $\tau$, the correlation time of direction fluctuations. The value of $\tau$ depends on the viscoelastic constants of the material under consideration and is, moreover, a function of the scattering angle. As the determination of the viscoelastic constants was not the primary aim of the present investigation, we kept the scattering angle fixed at a value of $5^{\circ}$, except 
for the measurements on a homeotropic sample, which were performed at a scattering angle of $20^{\circ}$. Knowledge of the correlation time $\tau$ and the average countrate of photocounts allowed us to compute the photocount distribution function $P(n, T)$ and the half-open time interval distribution $p_{\mathrm{f}}(t)$.

Both $P(n, T)$ and $p_{\mathrm{f}}(t)$ are related to the characteristic function $Q(s, t)$ defined as

$$
Q(s, t)=\left\langle\exp \left(-s \int_{t_{0}}^{t_{0}+t} \alpha I\left(t^{\prime}\right) \mathrm{d} t^{\prime}\right)\right\rangle,
$$

where the angle brackets denote ensemble averaging. In eq. (7) $I\left(t^{\prime}\right)$ is the light intensity incident on the detector at time $t^{\prime}$ and $\alpha I\left(t^{\prime}\right) \mathrm{d} t^{\prime}$ is the probability that a photocount will be generated between $t^{\prime}$ and $t^{\prime}+\mathrm{d} t^{\prime}$. In terms of $Q(s, t)$, $P(n, T)$ is given by (see e.g. ref. 5)

$$
P(n, T)=\left.\frac{(-)^{n}}{n !} \frac{\partial^{n}}{\partial s^{n}} Q(s, T)\right|_{s=1}
$$

and

$$
p_{\mathrm{f}}(t)=-\left.\frac{\partial}{\partial t} Q(s, t)\right|_{s=1} .
$$

For a mixture of Poisson light and Gaussian-Lorentz light, $Q(s, t)$ is of the form:

$$
Q(s, t)=Q_{\mathrm{P}}(s, t) Q_{\mathrm{GL}}(s, t)
$$

with

$$
Q_{\mathrm{P}}(s, t)=\exp \left(-s \bar{n}_{\mathrm{P}}\right)
$$

Here $\bar{n}_{\mathrm{P}}=\alpha\left\langle I_{\mathrm{P}}\right\rangle t$ is the average number of photocounts in an interval $t$ due to Poisson light with intensity $\left\langle I_{\mathrm{p}}\right\rangle$, and

$$
Q_{\mathrm{GL}}(s, t)=\exp (t / \tau)\left\{\cosh (z)+\frac{1}{2}(t /(z \tau)+(z \tau) / t) \sinh (z)\right\}^{-1},
$$

where $z=\left((t / \tau)^{2}+2(t / \tau) \bar{n}_{\mathrm{GL}} s\right)^{1 / 2}$. Here $\tau$ is the correlation time of the Gaussian-Lorentz process and $\bar{n}_{\mathrm{GL}}=\alpha\left\langle I_{\mathrm{GL}}\right\rangle t$ is the average number of photocounts in an interval $t$ due to Gaussian-Lorentz light. The total intensity is 
$\langle I\rangle=\left\langle I_{\mathrm{p}}\right\rangle+\left\langle I_{\mathrm{GL}}\right\rangle$ and, in the present case, $F_{\mathrm{P}} \equiv\left\langle I_{\mathrm{p}}\right\rangle /\left\langle I_{\mathrm{GL}}\right\rangle \ll 1$. As $\langle I\rangle$ and $\tau$ are both determined independently, the only adjustable parameter in the expressions for $P(n, T)$ and $p_{\mathrm{f}}(t)$ is $F_{\mathrm{P}}$, the fraction of uncorrelated photocounts. The value for $\langle I\rangle$ used in evaluating $P(n, T)$ and $p_{f}(t)$ has been corrected for deadtime and afterpulsing effects. Hence the computed $P(n, T)$ and $p_{\mathrm{f}}(t)$ are the "ideal" quantities. Before comparing these with the experimental data we take deadtime and afterpulsing effects into account, as indicated in eq. (3). Finally, we compare the computed $P(n, T)$ and $p_{t}(t)$ with the experimental ones. To obtain a quantitative measure for possible deviations from Gauss-Lorentz behavior, we computed $\chi^{2}$ for both $P(n, T)$ and $p_{f}(t)$ :

$$
\chi_{\mathrm{PD}}^{2}=\{1 /(M-2)\} \sum_{n=0}^{M}\left(P_{\mathrm{E}}(n, T)-P_{\mathrm{C}}(n, T)\right)^{2} / \sigma^{2}(n),
$$

where PD refers to the photocount Probability Distribution and the subscripts $E$ and $\mathrm{C}$ refer to experimental and computed, respectively, $M$ is the largest number of counts observed in interval $T$ during a given run and $\sigma^{2}(n)=$ $P_{\mathrm{C}}(n, T)\left(1-P_{\mathrm{C}}(n, T)\right) / N$, where $N$ is the total number of samples taken. Similarly, $\chi^{2}$ for $p_{f}(t)$ is defined as:

$$
\chi_{p_{\mathrm{f}}}^{2}=\left\{1 /\left(N_{\mathrm{U}}-N_{\mathrm{L}}-1\right)\right\} \sum_{i=N_{\mathrm{L}}}^{N_{\mathrm{U}}}\left(p_{\mathrm{f}}^{\mathrm{E}}(i \Delta t)-p_{\mathrm{f}}^{\mathrm{C}}(i \Delta t)\right)^{2} / \sigma^{2}(i) .
$$

Here $N_{\mathrm{U}}$ and $N_{\mathrm{L}}$ refer to the upper and lower limits on the index $i$. In eq. (14) $\Delta t$ is the time resolution with which the interval distribution is measured. The time range over which a measurement extends is determined by the setting of the time-to-amplitude converter. The longest time interval is $t_{\mathrm{MAX}}=N_{\mathrm{U}} \Delta t$. The shortest interval $t_{\text {MIN }}=N_{\mathrm{L}} \Delta t$ would ideally be zero but is in practice determined by the stability of the time-to-amplitude converter/pulse-height analysis system. Typically, to eliminate the effect of noise and jitter, we choose $t_{\mathrm{MIN}} \approx 0.01 t_{\mathrm{MAX}}$. $p_{\mathrm{f}}^{\mathrm{E}}$ and $p_{\mathrm{f}}^{\mathrm{C}}$ in eq. (1.4) refer to the measured and computed half-open interval distributions. The estimated error in $p_{f}(i \Delta t), \sigma(i)$, is given by $\sigma^{2}(i)=$ $p_{\mathrm{f}}^{\mathrm{C}}(i \Delta t)\left(1-p_{\mathrm{f}}^{\mathrm{C}}(i \Delta t) / N\right.$, where $N$ is the total number of intervals measured.

Fig. 1 shows a typical measurement of $P(n, T)$ and the fit to the experimental data. In fig. 2, the corresponding measurement of $p_{t}(t)$ is shown. The curve through the data points is characterized by the same parameters $\left(\langle I\rangle, \tau\right.$ and $\left.F_{\mathrm{P}}\right)$ as the curve in fig. 1. Figs. 3-6 show the temperature dependence of $\chi_{\mathrm{PD}}^{2}$ and $\chi_{p_{\mathrm{f}}}^{2}$ as a function of $T / T_{\mathrm{NI}}$ for DIBAB (fig. 3: planar, fig. 4: homeotropic), MBBA (fig. 5) and 5-CB (fig. 6). In no case is there any evidence for systematic deviations of $\chi^{2}$ from the value 1 . Hence we must conclude that the photon statistics of laser light scattered by nematic director fluctuations is compatible with the assumption that the time cvolution of director fluctuations is a 

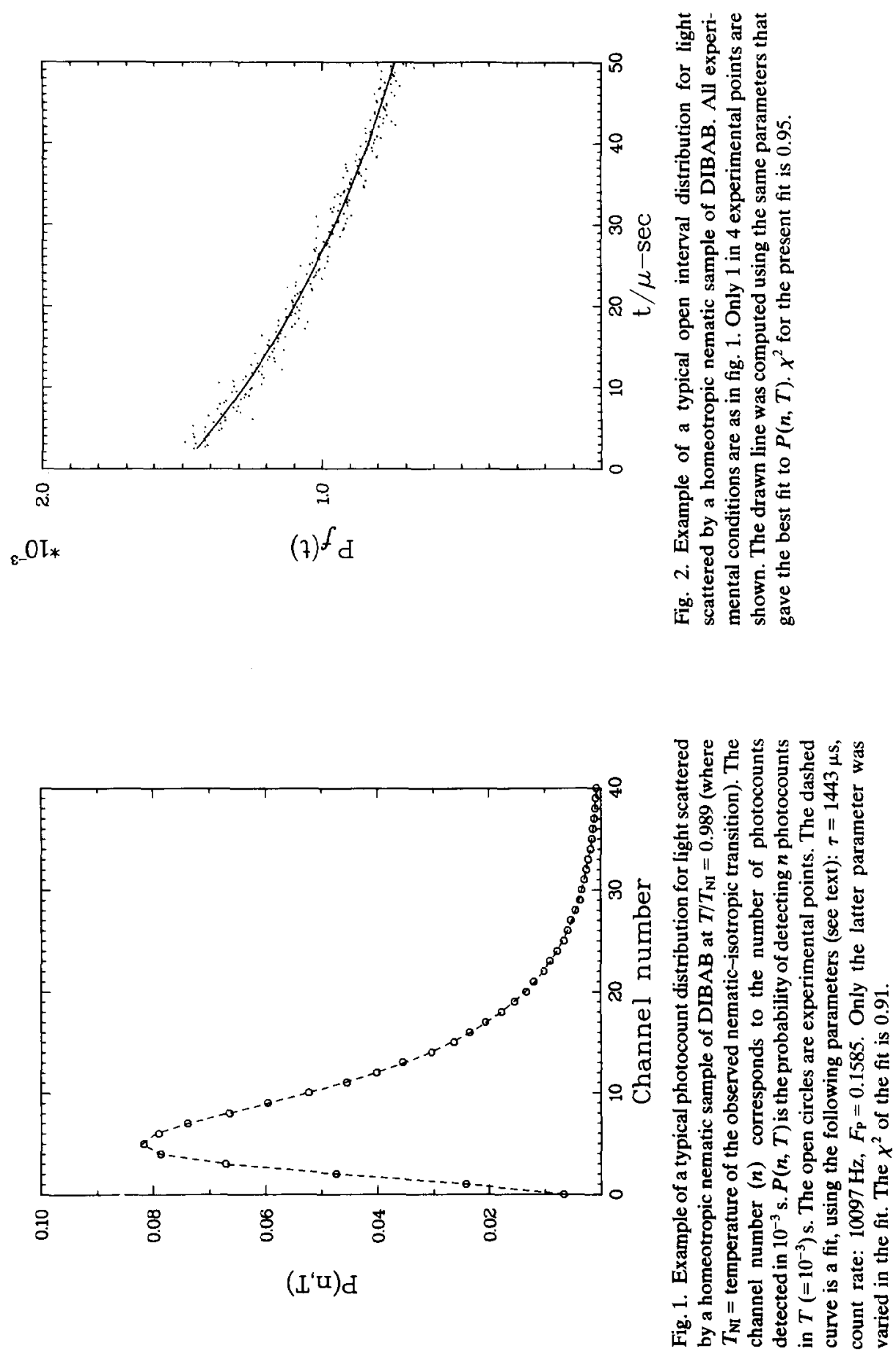

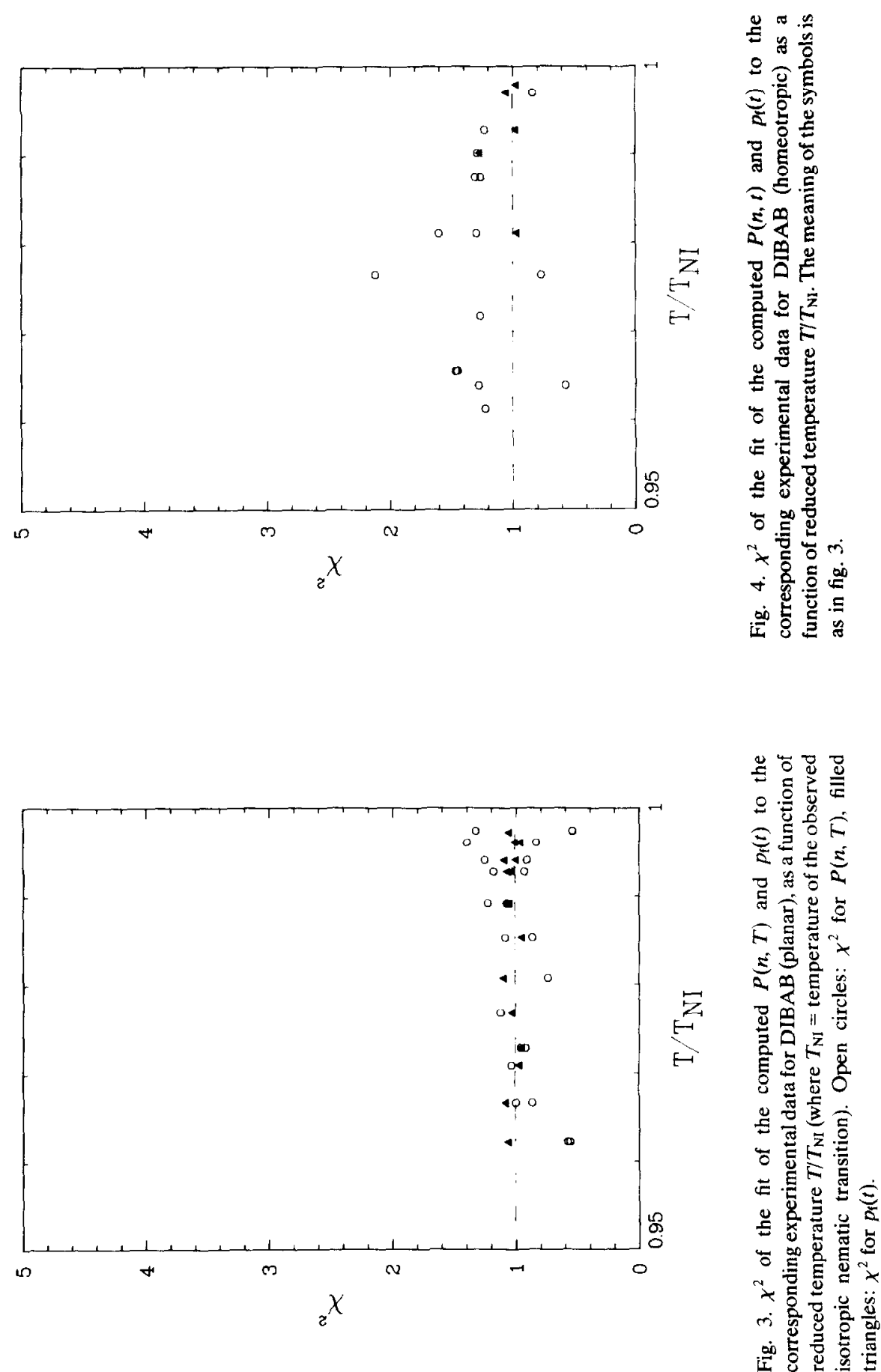


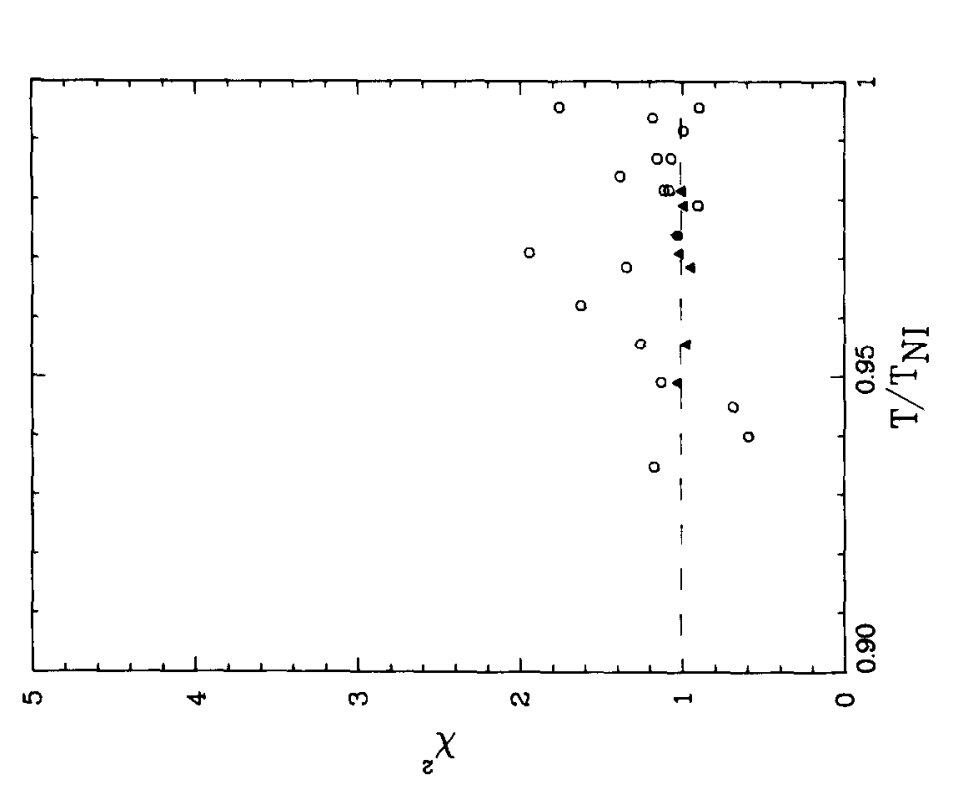

㝳

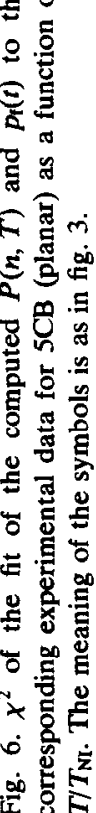

袺

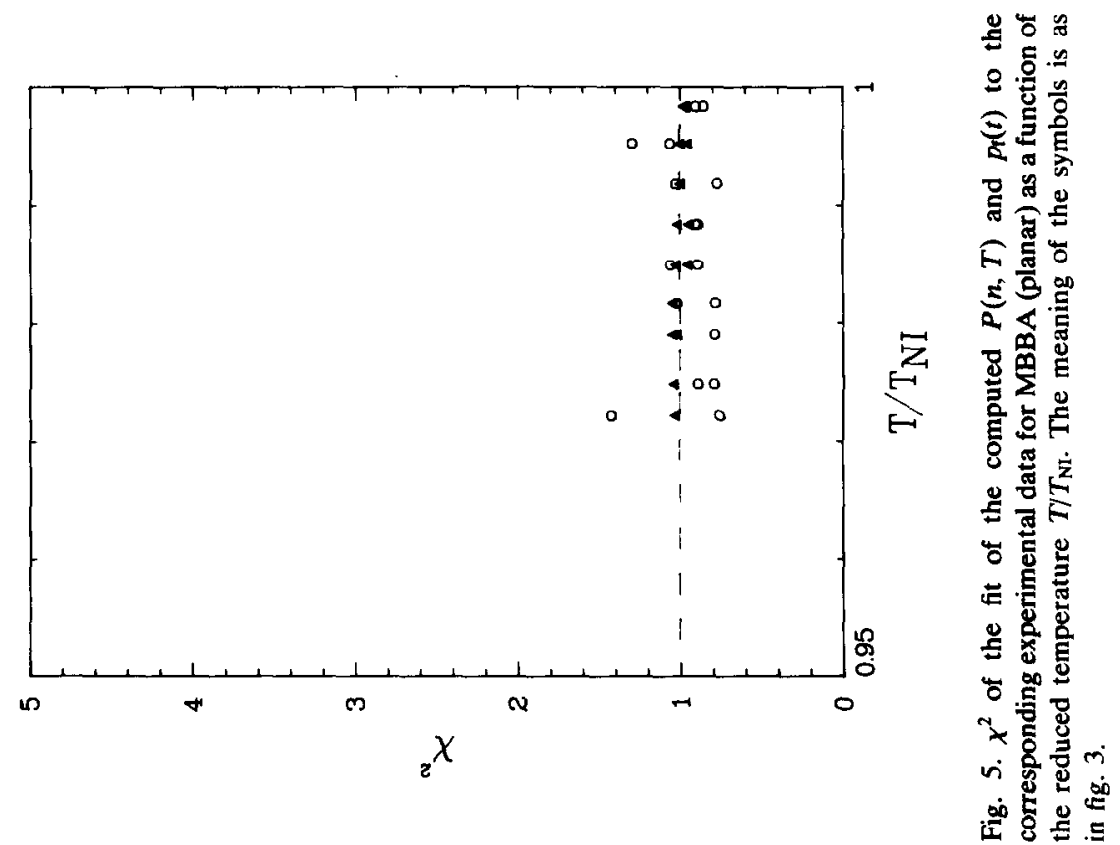


Gaussian-Lorentz process. We should add that the value of $\chi^{2}$, in particular for the photocount distribution $P(n, T)$, is very sensitive to the value of $F_{\mathrm{P}}$. Changing $F_{\mathrm{P}}$ by less than $1 \%$ may double $\chi^{2}$, even though such a change would have a barely perceptible effect on the fit in fig. 1 . Hence one should be careful not to judge the quality of the fit from its visual appearance alone. Finally it should be mentioned that the fact that we observe no deviations from Gaussian behavior does not rule out non-Gaussian behavior of the director fluctuations. The reason is that the scattered light is always modulated by a number of director modes with almost equal wave vectors and therefore almost equal relaxation times. If all these modes are uncorrelated, the central limit theorem tells us that we should observe Gaussian statistics of the scattered light, even if the individual modes are non-Gaussian. Non-Gaussian behavior could be observed if non-linear coupling introduced pronounced correlations between director modes with different wave vectors. Our experiments seem to rule out the latter possibility.

\section{References}

1) P.G. de Gennes, The Physics of Liquid Crystals (Oxford Univ. Press, New York, 1979).

2) Orsay Liquid Crystal Group, J. Chem. Phys. 51 (1969) 816.

3) J.P. van der Meulen and R.J.J. Zijlstra, J. de Physique 45 (1984) 107.

4) E. Jakeman, C.J. Oliver and E.R. Pike, J. Phys. A3 (1970) L45.

5) B. Saleh, Photoelectron Statistics (Springer, Berlin, 1978).

6) P. Tartaglia and S.H. Chen, J. Chem. Phys. 58 (1973) 4389.

7) W.W. Beens, Thesis, Univ. of Groningen, 1984.

8) S.H. Chem, C.C. Lai, J. Rouch and P. Tartaglia, J. Stat. Phys. 30 (1983) 699.

9) J. van Der Veen, W.H. de Jeu, A.H. Grobben and J. Boven, Mol. Cryst. Liq. Cryst. 17 (1972) 291.

10) C.L. Metha, in: Progress in Optics, vol. 8, E. Wolf, ed. (North-Holland, Amsterdam, 1970), p. 373. 